\title{
Self-Assembled Triply Periodic Minimal Surfaces as moulds for Photonic Band Gap Materials
}

\author{
L. Martín-Moreno*, F. J. García-Vidal ${ }^{\dagger}$ and A. M. Somoza ${ }^{\ddagger}$ \\ * Departamento de Física de la Materia Condensada, ICMA-Consejo Superior de Investigaciones Científicas, Universidad de \\ Zaragoza, Zaragoza 50015, Spain. \\ † Departamento de Física Teórica de la Materia Condensada, Universidad Autónoma de Madrid, Cantoblanco, 28049, \\ Madrid, Spain. \\ ¥ Departamento de Física. Universidad de Murcia. Aptdo. 4021, Murcia E-30080,Spain.
}

\begin{abstract}
We propose systems with structures defined by self-assembled triply periodic minimal surfaces (STPMS) as candidates for photonic bandgap materials. To support our proposal we have calculated the photonic bands for different STPMS and we have found that, at least, the double diamond and gyroid structures present full photonic bandgaps. Given the great variety of systems which crystalize in these structures, the diversity of possible materials that form them and the range of lattice constants they present, the construction of photonic bandgap materials with gaps in the visible range may be presently within reach.
\end{abstract}

PACS numbers: 42.70.Qs, 41.20.Jb, 81.05.Ys, 81.05.Qk

A three-dimensional (3D) photonic bandgap material (PBGM) is a periodic dielectric system with an absolute frequency gap for electromagnetic (EM) waves [i]. The opening of photonic gaps is a delicate balance between both refraction index contrast and topology of the underlying lattice. Since its proposal [2] [3] and first construc-

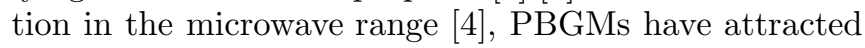
much attention due to their many remarkable applications [5], especially for gaps in the visible range. However, material engineering of 3D periodic systems structured at the relevant length scale for optical gaps (hundreds of $n m$ ) is facing technological problems [6]. Another promising strategy is mesoscopic self-assembly: in this way partial bandgaps have been reported in 3D periodic structures synthesized from submicron colloidal particles [7] [8]. Within this route, an alternative idea has apparently been overlooked: the use of self-assembled triply periodic minimal surfaces (STPMS) as natural moulds to construct optical PBGMs. STPMS are present in different systems like the periodic phases found in diblockcopolymers [9 and lipid/water systems [10]. Recently, the predicted 11] periodic phases in microemulsions have also been observed [12].

All the binary and ternary systems mentioned above have the common property of being self-organized at a supramolecular level, with morphologies mainly dictated by the shape of internal interfaces separating different domains. In $\mathrm{AB}$ diblock-copolymers these internal interfaces are formed by the chemical bonds between the $\mathrm{A}$ and B blocks, whereas in lipid/water systems or in microemulsions (of for example water and oil) the amphiphilic molecules form a monolayer interface that fully separates the polar compounds (water and amphiphilic heads) from covalent ones (oil and amphiphilic tails). From the physical point of view, the dominant factor that controls the shape of these internal interfaces is minimization of its surface free energy. This last condition is essentially equivalent to area-minimisation with fixed volume fractions and leads to the formation of constant mean curvature interfaces. When the different domains have approximately equal volume fractions, the mean curvature is zero. This basically explains why and when zero mean curvature interfaces are present in these systems. These kind of surfaces are known by mathematicians as minimal surfaces and, already in the last century [13], it was found that some minimal surfaces can be joined together forming periodic structures in all three spatial directions. Subsequentently, it was discovered [10] that minimal surfaces appear in a variety of real systems occurring in biology and materials science.

Presently known systems with STPMS have three appealing characteristics for an optical PBGM: i) Their lattice constants can be tuned by varying their constituents: real systems with STPMS have lattice constants ranging from one to hundreds of $\mathrm{nm}$. ii) Their morphologies are bicontinuous with cubic symmetry, which is known [1] to be more appropriate to produce photonic bandgaps than the close-packed morphologies resulting in self-assembled colloidal crystals. iii) Flexibility in the composition, either by the appropriate choice of the constituents, or by modifications through selective chemical reactions, may provide the adequate refraction index contrast.

However, although promising, these characteristics are not enough to secure the opening of photonic bandgaps: as previously mentioned, topology also plays a crucial role. So, in order to support our proposal of STPMS as possible PBGM, we have studied theoretically which architectures, if any, among the most commonly observed STPMS, develop bandgaps. We have performed calculations for the structures defined by three different minimal surfaces: simple cubic $(\mathrm{P})$, gyroid $(\mathrm{G})$ and double diamond (D). Our computational scheme has two steps: (i) generation of the different structures and (ii) calculation of the photonic bands for each one of them. We have restricted ourselves to systems with two different components of equal volume fraction domains, characterized by their refraction indexes $n_{1}$ and $n_{2}$ (for definiteness we take $\left.n_{1}<n_{2}\right)$, separated by a minimal surface.

There are several mathematical techniques (Weierstrass parameterization, finite element methods, ...) [14] for the generation of triply periodic minimal surfaces. Based on physical grounds, it is expected that these periodic surfaces could be generated via minimisation of a Landau-Ginzburg free energy functional [15], result that 
has been proved rigorously [16]. We have chosen this last approach, finding extremal configurations of the functional by solving numerically the Cahn-Hilliard equation [17]. This equation resembles the time evolution of the local concentration $c(\vec{r})$ in the dynamical process of phase separation in binary mixtures of polymers and alloys. The local concentration is forced to verify a continuity equation, which guarantees fixed volume fractions during the full dynamical process. As the final result we obtain the stable (or metastable) time-evolved $c(\vec{r})$ evaluated at a 3D regular grid of $N^{3}$ points covering the unit cell. Different triply periodic minimal surfaces, defined as the interface between both constituents, are obtained for different initial configurations of $c(\vec{r})$; known solutions are recovered by initializing $c(\vec{r})$ near to the expected result. In Figs. 1a, 1b and 1c we show, respectively, the P, G and D structures obtained via this method.

As regards to the photonic band calculation, we have used a finite difference time domain (FDTD) method 18 . This method requires a computational time which scales linearly with the number of mesh points inside the unit cell and, therefore, is specially suitable for systems with a dielectric constant that changes rapidly in space, like the ones we are studying. Basically, in the FDTD method an arbitrary initial EM wave with a wavenumber $\vec{k}$ is allowed to evolve both in time and space governed by a discrete version of Maxwell equations. After a certain run time, long enough to have good spectral resolution, the frequencies $\omega(\vec{k})$ of EM eigenmodes in the structure are obtained by Fourier transforming the time-propagated EM fields. For each structure we have performed calculations for values of $N$ ranging from $N=14$ to $N=40$. We have checked that the results obtained converge rapidly with $N$, which gives us confidence on the quality and consistency of both structural and photonic calculations.

It is worth pointing out that, although we are thinking on applications in the optical regime, our results are valid for any lattice constant due to the scaling properties of the macroscopic Maxwell equations [1]. Gaps can be characterised by two quantities: the wavelength at the midgap frequency $\left(\lambda_{0}\right)$ in units of the lattice constant $(a)$, times the smallest refraction index $\left(n_{1}\right), \lambda_{0} /\left(n_{1} a\right)$, and the "figure of merit", $f$, defined as the quotient between the frequency width of the gap and the midgap frequency. $f$ is, at the same time, a measure of the gap, of the reduction of transmission of EM waves though a finite number of layers, and of the robustness of the gap to small deviations from uniformity of the lattice constant throughout the crystal.

We have calculated the photonic bands as a function of the index of refraction contrast $n=n_{2} / n_{1}$, for each one of the cited $3 \mathrm{D}$ periodic structures. For the $\mathrm{P}$ structure we have not found photonic gap for any $n$. This result is in keeping with photonic band calculations for the, different but related, system of dielectric spheres assembled in a simple cubic lattice. Inset of Fig. 1a shows the low-frequency photonic bands for the $\mathrm{P}$ structure for a representative $n=\sqrt{13}$, showing the lack of gap in the spectrum due to some degeneracies in points of high symmetry. However, and this is the main outcome of our calculations, we do find gaps for the G and D structures, provided there is a minimum refraction index contrast be- tween the two materials. This can be clearly seen in the insets of Figs. 1b and 1c where the low-frequency photonic bands for the $\mathrm{G}$ and $\mathrm{D}$ structures are shown, for the same representative refraction index contrast $n=\sqrt{13}$. Fig. 2a summarizes our results for the G structure, showing the dependence of both $\lambda_{0} /\left(n_{1} a\right)$ and $f$ with $n$. Fig. $2 \mathrm{~b}$ shows the same quantities as Fig. 2a, this time for the D structure. Notice that, due to the fact that midgap wavelengths are approximately twice as large as lattice constants (an even larger for large $n$ or large $n_{1}$ ), photonic bandgaps in the optical regime are expected for lattice constants of $\approx 200-350 \mathrm{~nm}$ or even smaller.

It is noteworthy that not only there is a minimum value of $n$ for the appearance of photonic gaps, but also the quality factor $f$ increases with $n$ both for the $\mathrm{G}$ and $\mathrm{D}$ structures, thus making large refraction index contrast a desirable feature. The great flexibility in the manipulation of known systems with STPMS may provide routes to obtain the desired refraction index contrast. For example, in $\mathrm{AB}$ diblock copolymers, the optical properties can be tailored modifying the $\mathrm{A}$ and $\mathrm{B}$ monomers, triblock copolymers providing even more flexibility. In lyotropic systems and microemulsions their liquid nature turns into an advantage. One of the liquids may be solidified (through freezing or polymerization) and the other one extracted. Moreover, the domains of one of the constituents may be used as a template for many different chemical reactions. For instance, in this way cubic structures of silica with remarkable periodicity have been already obtained [19]. The reported lattice constant was about $10 \mathrm{~nm}$ but it might be possible to increase this value, as pore sizes larger than $100 \mathrm{~nm}$ have been found in disordered silica mesostructures [20]. Extraction of the surfactant and substitution by another material with a large dielectric constant may lead to a PBGM in the ultraviolet regime. Even more interestingly, materials presenting STPMS have been used as hosts for synthesizing semiconductors with very large refraction index [21. Based on all these, we believe that the construction of 3D PBGMs in the ultraviolet and optical ranges based on STPMS could be built using present day chemistry.

Acknowledgments. We acknowledge useful discussions with Prof. R. Strey and Prof. P. Tarazona, and Prof. J. B. Pendry and Dr. A.J. Ward for providing us with the FDTD code [18] prior to its publication.

[1] J. Joannopoulos, R. Meade and J. Winn Photonic Crystals (Princeton Press, Princeton, NJ, 1995).

[2] E. Yablonovitch. Phys. Rev. Lett. 58, 2059-2062 (1987).

[3] S. John. Phys. Rev. Lett. 58, 2486-2489 (1987).

[4] E. Yablonovitch, T. J. Gmitter and K. M. Leung. Phys. Rev. Lett. 67, 2295-2298 (1991).

[5] J. D. Joannopoulos, P. R. Villeneuve and S. Fan. Nature 386, 143 (1997).

[6] The manufactured 3D-PBGM with the smallest midgap wavelength $(12 \mu \mathrm{m})$ has been recently constructed: S. Y. Lin et al.. Nature 394, 251-253 (1998). 
[7] H. Miguez et al. Appl. Phys. Lett. 71, 1148-1150 (1997).

[8] J. E. G. J. Wijnhoven and W. L. Vos. Science 281, 802804 (1998).

[9] E. L. Thomas, D. M. Anderson, C. Henkee and D. Hofman. Nature 334, 598-601 (1988).

[10] V. Luzatti and P. A. Spegt. Nature 215, 701-704 (1967).

[11] L. E. Scriven. Nature 262, 123-125 (1976).

[12] R. Strey. (personal communication, 1998).

[13] H. A. Schwarz. Gesammelte Mathematische Abhhandlungen, (Springer, 1890).

[14] J. Klinowski, A. L. Mackay and H. Terrones. Phil. Trans. R. Soc. Lond. A 354, 1975-1987 (1996).

[15] W. T. Góźdź and R. Hołyst. Phys. Rev E 54, 5012-5027 (1996).

[16] L. A. Caffarelli and A. Córdoba. Differential and Integral Equations 6, 1-13 (1993).

[17] J. A. Bray. Adv. Phys. 43, 357-459 (1994).

[18] A. J. Ward and J. B. Pendry. Comp. Phys. Comm. 112, 23-41 (1998).

[19] A. Monnier et al. Science 261, 1299-1303 (1993).

[20] K. M. Mcgrath et al. Science 277, 552-556 (1997).

[21] J. P. Yang, S. B. Qadri and B. R. Ratna. J. Phys. Chem. 100, 17255-17259 (1996).

\section{Figure Captions}

Figure 1. Three unit cells for the different triply periodic minimal surfaces analyzed in this paper: (a) simple cubic $\mathrm{P},(\mathbf{b})$ gyroid $\mathrm{G}$ and (c) double diamond $\mathrm{D}$. To the right of each structure, the corresponding photonic band structure is shown, for the representative case in which the two materials separated by the minimal surface have a refraction index contrast $n=\sqrt{13}$. The wave vector varies across the simple cubic Brillouin zone defined by the high-symmetry points: $\mathrm{G}(0,0,0), \mathrm{X}\left(0,0, \frac{\pi}{a}\right), \mathrm{M}\left(0, \frac{\pi}{a}, \frac{\pi}{a}\right)$ and $\mathrm{R}\left(\frac{\pi}{a}, \frac{\pi}{a}, \frac{\pi}{a}\right), a$ being the lattice constant of the system. EM frequency is in units of $\frac{2 \pi c}{n_{1} a}$ where $\frac{c}{n_{1}}$ is the light velocity in the lowest refraction index material. Note the appearance of a complete photonic bandgap, highlighted in yellow, in the $\mathrm{G}$ and $\mathrm{D}$ band structures.

Figure 2. Quality factor, $f$ (full line) and midgap wavelength $\lambda_{0}$ (dashed line) as a function of the refraction index contrast for the (a) gyroid and (b) double diamond structures. $f$ is the quotient between the gap width and the midgap frequency. $\lambda_{0}$ is in units of the lattice constant $a$ times the lowest refraction index $n_{1}$. For both structures there is a minimum refraction index contrast to open a bandgap, this value being $n \approx 2.5$ for the $\mathrm{G}$ case and $n \approx 2$ for the $\mathrm{D}$ one. 
This figure "fig1.gif" is available in "gif" format from: http://arxiv.org/ps/cond-mat/9810299v1 


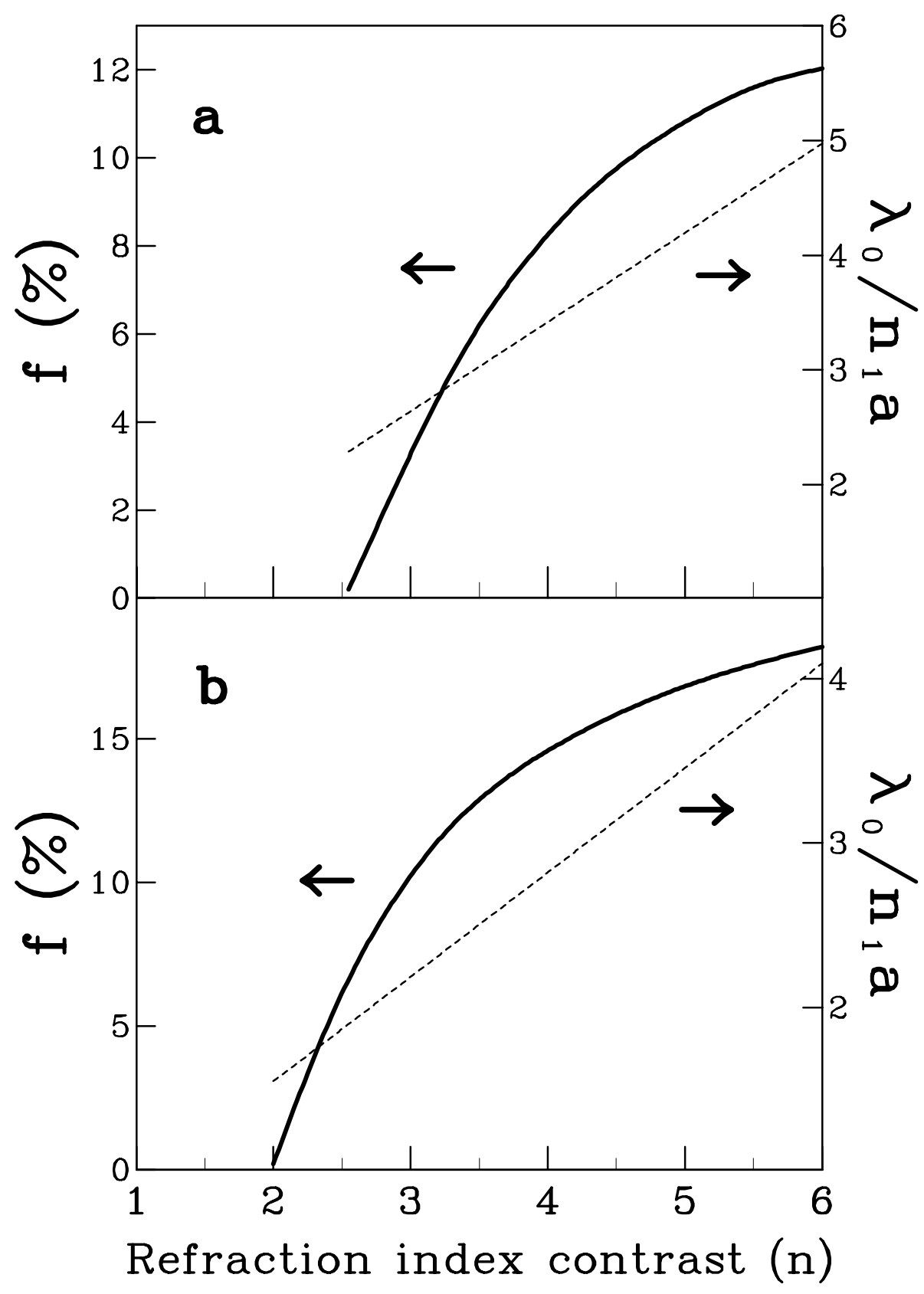

